\title{
Fatores associados à qualidade de vida relacionada à saúde em adolescentes escolares com excesso de peso na pandemia de COVID-19
}

\author{
Factors associated with health-related quality of life in overweight schoolchildren in the COVIID-19 \\ pandemic
}

Factores asociados a la calidad de vida relacionada con la salud en escolares con sobrepeso en la pandemia COVID-19

\section{Resumo}

Objetivo: Avaliar os fatores associados à qualidade de vida relacionada à saúde em adolescentes escolares durante a pandemia de COVID-19. Método: Estudo transversal, realizado com 87 adolescentes de escola municipal de uma cidade do centro-oeste mineiro. Escala KIDSCREEN 52 foi utilizada para avaliar a Qualidade de Vida Relacionada à Saúde. Foram obtidos peso e altura autorrelatados, nível socioeconômico, consumo alimentar e nível de atividade física. Regressão linear múltipla foi realizada. Os dados foram analisados pelo programa estatístico Statistical Package for Social Sciences versão 21.0. Resultados: A prevalência de excesso de peso foi 32,2\% e 56,9\% dos adolescentes consumiram de três a cinco tipos de alimentos processados e/ou ultraprocessados no dia anterior à entrevista. Um percentual de 17,2\% dos participantes foi classificado como sedentário e insuficiemente ativo e participantes da classe social C 1 obtiveram maiores medianas no domínio autopercepção corporal. Maiores medianas do domínio estado emocional foram evidenciadas em adolescentes do sexo feminino e aqueles que consumiam alimentos processados e ultraprocessados. Os meninos apresentaram maiores medianas nos domínios saúde e atividade física, sentimentos e amigos e apoio social. A análise multivariada apontou que o aumento da idade e do índice de massa corporal (IMC) associou-se à diminuição da qualidade de vida relacionada à saúde global. Conclusão: Os resultados mostram a importância do aprimoramento de políticas públicas voltadas para a qualidade de vida dos adolescentes com foco na promoção da alimentação saudável e estímulo à atividade física, em especial, durante a pandemia de COVID-19.

Palavras-chave: Adolescente; Qualidade de vida; Estado nutricional; Obesidade; Saúde.

\footnotetext{
Abstract

Objective: To evaluate factors associated with Health-Related Quality of Life in adolescent schoolchildren during the COVID-19 pandemic. Method: Cross-sectional study, carried out with 87 adolescents from a municipal school in a
} 
city in the Midwest of Minas Gerais. The KIDSCREEN 52 scale was used to assess the Health-Related Quality of Life. Self-reported weight and height, socioeconomic level, food consumption and physical activity level were obtained. Multiple linear regression was performed. Data were analyzed using the statistical program Statistical Package for Social Sciences version 21.0. Results: The prevalence of overweight was 32.2\% and 56.9\% of the adolescents consumed three to five types of processed and/or ultra-processed foods the day before the interview. A percentage of $17.2 \%$ of the participants were classified as sedentary and insufficiently active, and participants from social class $\mathrm{C} 1$ had higher medians in the body self-perception domain. Higher medians of the emotional state domain were found in female adolescents and those who consumed processed and ultra-processed foods. Boys had higher medians in the health and physical activity, feelings and friends, and social support domains. The multivariate analysis showed that increasing age and body mass index (BMI) were associated with a decrease in quality of life related to global health. Conclusion: The results show the importance of improving public policies aimed at the quality of life of adolescents with a focus on promoting healthy eating and encouraging physical activity, especially during the COVID-19 pandemic.

Keywords: Adolescent; Quality of life; Nutritional Status; Obesity; Health.

\begin{abstract}
Resumen
Objetivo: evaluar los factores asociados a la calidad de vida relacionada con la salud en escolares adolescentes durante la pandemia de COVID-19. Método: Estudio transversal, realizado con 87 adolescentes de una escuela municipal de una ciudad del Medio Oeste de Minas Gerais. Para la evaluación de la Calidad de Vida Relacionada con la Salud se utilizó la escala KIDSCREEN 52. Se obtuvo el peso y la estatura autoinformados, el nivel socioeconómico, el consumo de alimentos y el nivel de actividad física. Se realizó regresión lineal múltiple. Los datos se analizaron mediante el programa estadístico Statistical Package for Social Sciences versión 21.0. Resultados: La prevalencia de sobrepeso fue de 32,2\% y el 56,9\% de los adolescentes consumieron de tres a cinco tipos de alimentos procesados y / o ultraprocesados el día anterior a la entrevista. Un porcentaje del 17,2\% de los participantes fueron clasificados como sedentarios e insuficientemente activos y los participantes de la clase social C 1 tenían medianas más altas en el dominio de autopercepción corporal. Se evidenciaron medianas más altas del dominio del estado emocional en mujeres adolescentes y aquellas que consumían alimentos procesados y ultraprocesados. Los niños tenían medianas más altas en los dominios de salud y actividad física, sentimientos y amigos y apoyo social. El análisis multivariado mostró que el aumento de la edad y el índice de masa corporal (IMC) se asociaron con una disminución de la calidad de vida relacionada con la salud global. Conclusión: Los resultados muestran la importancia de mejorar las políticas públicas dirigidas a la calidad de vida de los adolescentes con un enfoque en la promoción de una alimentación saludable y el fomento de la actividad física, especialmente durante la pandemia de COVID-19.
\end{abstract}

Palabras clave: Adolescente; Calidad de vida; Estados nutricionales; Obesidad; Salud.

\title{
1. Introdução
}

O excesso de peso entre adolescentes brasileiros tornou-se um sério problema de saúde pública e apresenta tendência de crescimento (Conde et al., 2018). Em todo o mundo, o número de crianças e adolescentes com obesidade aumentou mais de 10 vezes nas últimas décadas, passando de 11 milhões em 1975 para 124 milhões em 2016 (NCD Risk Factor Collaboration, 2017). Cerca de $22 \%$ da população brasileira entre cinco e 19 anos de idade apresenta excesso de peso (Guedes \& Mello, 2021). A Pesquisa Nacional de Saúde do Escolar (PeNSE) mostrou que de 23,7\% dos estudantes entre 13 e 17 anos tinham sobrepeso $(15,9 \%)$ ou obesidade $(7,8 \%)$ em 2015 no Brasil, o que correspondia a um total aproximado de 3,1 milhões de jovens (Instituto Brasileiro de Geografia e Estatística, 2015).

A obesidade implica na ocorrência de múltiplas repercussões negativas em diversos aspectos da saúde de adolescentes. Dentre as principais repercussões, citam-se os prejuízos físicos e psicológicos, como depressão, ansiedade, distúrbios do sono, baixa autoestima, imagem corporal distorcida, comprometimento no rendimento escolar, dificuldades de relacionamento e complicações metabólicas, como o desenvolvimento de doenças crônicas não transmissíveis (DCNT) (Cunha et al., 2018), o que, provavelmente, influencia a qualidade de vida relacionada à saúde (QVRS).

A Qualidade de Vida Relacionada à Saúde (QVRS) é um conceito que envolve três principais dimensões da saúde, sendo física, psicológica e social, que somadas podem indicar um resultado que representa a saúde geral (Bacchini et al., 2017; Gunawardana et al., 2021). A literatura aponta determinantes da QVRS em adolescentes, como nível socioeconômico, alimentação, estado nutricional, nível de atividade física, autoimagem, tempo de tela, sintomas depressivos e suporte social (Nascimento et al., 2016; Pussieldi; Silva; Pereira, 2018; D’avila et al., 2019; Liu et al., 2019; Zhao et al., 2018; Otto et al., 
2017). Investigações têm verificado que o excesso de peso também tem o potencial de influenciar a QVRS, porém os resultados não são consensuais (Baile et al., 2020; Yi et al.,, 2019; Peres-Sousa et al., 2018; Arruda et al., 2020). Além disso, os fatores que se associam à QVRS em adolescentes certamente podem ser potencializados pela pandemia de COVID-19, a maior catástrofe mundial da contemporaneidade (Gaudenzi, 2021). De fato, as medidas de mitigação da doença, como distanciamento social e fechamento de escolas, têm impactado a saúde do adolescente, com surgimento e agravamento de problemas de saúde mental e aumento da violência intradomiciliar (Oliveira et al., 2021).

A presente investigação tem o potencial de trazer indicadores para a literatura acerca dos determinantes da QVRS em adolescentes, com foco especial no excesso de peso e no contexto da pandemia de COVID-19. Os resultados poderão contribuir para o aprimoramento de políticas públicas de atenção à saúde do adolescente. Assim, o objetivo desta pesquisa consiste em avaliar os fatores associados à qualidade de vida relacionada à saúde em adolescentes escolares durante a pandemia de COVID-19.

\section{Metodologia}

\section{Desenho e local do estudo}

Estudo transversal, realizado em uma escola municipal situada na zona urbana de uma cidade do centro-oeste mineiro. A pesquisa ocorreu no mês de março de 2021, período este em que os adolescentes encontravam-se em isolamento social, com aulas remotas. Este estudo foi elaborado conforme as diretrizes metodológicas do Strengthening the Reporting of Observational studies in Epidemiology (STROBE) (Cuschieri, 2019).

\section{População do estudo}

A população do estudo foi constituída pelo universo de adolescentes matriculados na escola no ano de 2021, perfazendo um total de 89 participantes. O critério de inclusão consistiu em o pai ou responsável possuir telefone celular com o aplicativo WhatsApp instalado. Os critérios de exclusão da pesquisa envolveram adolescentes diagnosticados com doença genética ou outra patologia que pudesse interferir no desfecho QVRS e não se conseguir contato telefônico. Dois adolescentes foram excluídos devido aos pais não atenderem às chamadas telefônicas, portanto, a amostra final do estudo foi de 87 participantes.

\section{Recrutamento e Aspectos Éticos}

Foi obtida da Secretaria Municipal de Educação (SEMED) uma lista com os contatos telefônicos dos pais ou responsáveis pelos adolescentes. Foi realizado contato com pais ou responsáveis, através de ligação telefônica, para a explanação do projeto. Nesse momento, eles tiveram a oportunidade de sanar qualquer dúvida referente à pesquisa. Realizadas todas as informações sobre a presente investigação, os pesquisadores efetuaram o convite para a autorização da participação do adolescente. Após o aceite, foi enviado o termo de consentimento livre esclarecido (TCLE) via WhatsApp. O mesmo procedimento foi feito com o adolescente e, ao final, obtido o Termo de Assentimento Livre e Esclarecido (TALE). O projeto foi aprovado pelo Comitê de Ética em Pesquisa (CEP) da Universidade Federal de São João Del Rei, com o parecer consubstanciado número 4.208.094.

\section{Coleta de dados e Instrumentos de coleta}

Qualidade de vida relacionada à saúde

Para avaliar a QVRS, utilizou-se a Escala KIDSCREEN 52, versão brasileira, adaptada e validada por Guedes \& Guedes (2011). Esta escala é constituída por 52 itens e dez dimensões que descrevem a QVRS, sendo: Saúde e Atividade 
Física; Sentimentos; Estado de Humor Geral; Autopercepção Corporal; Tempo Livre; Família, Ambiente Familiar e Vizinhança; Questões Econômicas; Amigos; Ambiente Escolar e Aprendizagem e Provocação (Bullying). Nas dimensões, cada item é pontuado numa escala tipo Likert que varia entre 1 = Nada/Nunca, 2 = Um Pouco/ Quase Nunca; $3=$ Moderadamente/ Algumas vezes; 4 = Muito/Quase sempre; 5 = Muitíssimo/Sempre, sendo que quanto mais elevada for à pontuação, maior a percepção da qualidade de vida pelas crianças e adolescentes.

Na dimensão "Provocação (Bullying)" a pontuação foi invertida, apontando valores elevados na qualidade de vida se os sentimentos negativos forem raros. Uma pontuação alta implica sentir-se atormentado, provocado e rejeitado pelos seus pares e uma pontuação baixa revela não se sentir provocado, sentir-se respeitado e aceito pelos pares. Após somatório dos diferentes itens pertencentes a cada dimensão, os resultados foram transformados em valores de zero a 100 através da seguinte fórmula (score bruto- score mínimo esperado) /amplitude) x 100. Dessa forma, foi calculado o score mediano de cada dimensão (Bica et al., 2020).

Os resultados foram agrupados por dimensões e apurou-se que um score de 50 é o valor mediano de cada dimensão. Desse modo, considerou-se que a percepção dos adolescentes sobre a QVRS foi positiva (adequada) quando apresentou scores superiores à mediana 50 e, pelo contrário, scores inferiores à mediana 50 representaram uma percepção negativa (inadequada) da QVRS (Bica et al., 2020).

\section{Avaliação do estado nutricional}

Para a determinação do IMC, utilizou-se o autorrelato do peso e da altura dos participantes. Essas informações foram lançadas no Software Who Anthro Plus que assume o padrão atual da Organização Mundial de Saúde (Lagares; Resende; Romano, 2019), estabelecendo como índice de avaliação o IMC por idade. Categorizou-se a variável em excesso de peso em "sim" quando o escore-z de IMC por idade foi > escore-z + 1, e "não" quando $\leq$ escore-z +1 .

\section{Nivel socioeconômico e dados demográficos}

O nível socioeconômico foi avaliado por meio do questionário de critérios de classificação da Associação Brasileira de Empresas de Pesquisa (ABEP) que se baseia no cômputo dos bens existentes no domicílio (eletrodomésticos e carros), presença de empregada doméstica mensalista, escolaridade do chefe da família e no acesso a serviços públicos. As categorias podem variar de A (nível mais elevado) até E (nível mais baixo), de acordo com a pontuação obtida (Associação Brasileira de Empresas de Pesquisa, 2015).

Os adolescentes foram caracterizados quanto ao sexo (masculino e feminino). A escolaridade dos adolescentes foi caracterizada em ano de matrícula (oitavo ou nono). A cor da pele foi autorrelatada pelos adolescentes e categorizada em: negra, parda e branca. A idade cronológica foi apresentada como variável contínua.

\section{Nível de atividade física}

O nível de atividade física dos adolescentes foi mensurado pelo International Physical Activity Questionnaire (IPAQ) versão curta, validado para adolescentes brasileiros. Entre os parâmetros avaliados pelo IPAQ, incluem-se dados sobre atividade física: duração (minutos por dia), frequência (dias por semana), intensidade e tipo de atividade, tendo a última semana como referência. Os adolescentes foram classificados conforme o consenso proposto pelo Centro Laboratório de Estudos de Aptidão Física de São Caetano do Sul sendo: Muito Ativo: $\geq 5$ dias/sem e $\geq 30$ minutos/sessão de atividade vigorosa, ou $\geq 3$ dias/sem e $\geq 20$ minutos/sessão de atividade vigorosa $+\geq 5$ dias/sem e $\geq 30$ minutos/sessão de atividade moderada e/ou caminhada; Ativo: $\geq 20$ minutos/sessão de atividade vigorosa $\geq 3$ dias/semana, e/ou $\geq 30$ minutos por sessão de atividade física moderada, ou caminhada $\geq 5$ dias/semana, e/ou $\geq 150$ minutos/semana de qualquer das atividades combinadas 
(vigorosa + moderada + caminhada) $\geq 5$ dias/semana; Irregularmente ativo: aquele que realiza atividade física, porém insuficiente para ser classificado como ativo, pois não cumpre as recomendações quanto à frequência ou duração, somando-se a frequência e a duração dos diferentes tipos de atividades (vigorosa + moderada + caminhada) (Centro Coordenador do IPAQ no Brasil, 2017).

\section{Consumo alimentar}

O consumo alimentar foi avaliado utilizando o Questionário Simplificado de Diversidade Alimentar (Sattamini, 2019), que consiste em 26 perguntas sobre o consumo (sim ou não no dia anterior), divididas em dois subgrupos, primeiro grupo sobre alimentos in natura e o segundo grupo alimentos ultraprocessados, independente da quantidade consumida, fornecendo-se, quando pertinente, exemplos de tipo ou marcas. Esta variável foi convertida em alimentos in natura ou minimamente processados e alimentos ultraprocessados, conforme estabelecido no Guia Alimentar para a População Brasileira (Ministério da Saúde, 2014). Após o levantamento dos dados obteve-se o somatório de quantos alimentos pertencentes a cada grupo foram consumidos pelos adolescentes no dia anterior a coleta de dados. Também foram avaliadas as horas de sono, característica emocional (calma, agitada, ansiosa), número de pessoas residentes na mesma casa.

\section{Análise dos dados}

Após o levantamento dos dados, estes foram tabulados em dupla entrada, em planilha do Epidata e, posteriormente, foram analisados pelo programa estatístico Statistical Package for Social Sciences (SPSS) versão 21.0. Variáveis categóricas foram descritas em percentuais e variáveis contínuas em média e desvio-padrão (DP) ou mediana e quartis, conforme a distribuição de normalidade dos dados.

Os desfechos de QVRS foram comparados entre os grupos com excesso de peso e sem excesso de peso, entre os sexos e entre atividade física utilizando o teste de Mann Whitney e a comparação entre os níveis socioeconômicos foi realizada através do teste de Kruskal Wallis. Foram realizadas correlações de Spearman para relacionar consumo de alimentos processados e ultraprocessados e desfecho qualidade de vida. A qualidade de vida global foi avaliada através da regressão linear multivariada com a variável QVRS e o estado nutricional. Foi utilizado o software G*Power para realizar o cálculo do poder, que variou de 0,05127 a 0,9508, o nível de significância adotado nesse trabalho é de 5\%.

\section{Resultados}

A maior parte dos participantes do estudo estavam matriculados no nono ano do ensino fundamental 54,0\% ( $\mathrm{n}=47$ ), apresentavam idade média de 13,5 anos e eram do sexo feminino 58,9\% $(\mathrm{n}=52)$. Com relação à cor da pele, $46 \%(\mathrm{n}=40)$ consideravam-se pardos e o número de pessoas residentes na mesma casa foi igual ou superior a cinco moradores para $75 \%$ $(\mathrm{n}=65)$ dos adolescentes. A maioria deles residia em área urbana $(96,6 \%)(\mathrm{n}=84)$.

Ao avaliar as horas de sono, $75 \%(\mathrm{n}=65)$ dos adolescentes informaram dormir por 11 horas ou mais, as medianas para estatura e peso foram de $160 \mathrm{~cm}$ e $54 \mathrm{~kg}$ respectivamente. No que tange ao estado nutricional, $32,2 \%$ dos adolescentes apresentavam excesso de peso. Com relação ao nível socioeconômico 59,8\% (n=52) foram classificados como $\mathrm{C}_{1}$ (23-28 pontos) que corresponde a uma renda média de 2705 reais. Não foram evidenciados os níveis $\mathrm{A}, \mathrm{B}_{1}$ e $\mathrm{B}_{2}$ neste estudo.

Quanto ao nível de atividade física, 17,2\% (n=15) eram sedentários ou insuficientemente ativos e quanto ao consumo alimentar, observou-se que 63,2\% (n=55), consumiam de seis a oito tipos de alimentos in-natura ou minimamente processados e 56,9\% (n=49) dos adolescentes consumiam de três a cinco tipos de alimentos ultraprocessados ao longo do dia. Na avaliação dos domínios da escala Kidscreen 52, segundo a percepção dos adolescentes, a QVRS é adequada, exceto no que se refere ao domínio estado emocional que foi baixa. 
Evidenciou-se diferenças nas medianas dos domínios da QVRS no que tange ao sexo. Os participantes do sexo masculino apresentaram maiores medianas que os do sexo feminino no domínio saúde e atividade física ( $\mathrm{p}=0,000)$. Os meninos também apresentaram maiores medianas no domínio sentimentos $(\mathrm{p}=0,031)$ e no domínio amigos e apoio social $(\mathrm{p}=$ 0,019) (Tabela 1). Sobre o domínio estado emocional, as participantes do sexo feminino tiveram maiores medianas $(\mathrm{p}=0,020)$. (Tabela 1).

Tabela 1 - Associação entre Qualidade de vida e sexo em adolescentes escolares durante o período do isolamento social, 2021.

\begin{tabular}{llll}
\hline \hline & \multicolumn{2}{l}{ Sexo $($ mediana $(\mathrm{Q} 1-\mathrm{Q} 3)$} & \\
\hline Dimensão & Feminino $(\mathrm{n}=52)$ & Masculino $(\mathrm{n}=35)$ & valor $\mathrm{p}^{*}$ \\
\hline Saúde Atividade Física & $42,5(35-58,8)$ & $70(50-75)$ & $\mathbf{0 , 0 0 0}$ \\
Autopercepção corporal & $50(40-60)$ & $40(40-60)$ & 0,067 \\
Autonomia e Tempo Livre & $80(70-88,8)$ & $85(65-95)$ & 0,439 \\
Sentimentos & $83,3(71,9-91,7)$ & $95,8(75-100)$ & $\mathbf{0 , 0 3 1}$ \\
Estado Emocional & $33,3(17,7-45,8)$ & $25(4,2-33,3)$ & $\mathbf{0 , 0 2 0}$ \\
Família e Ambiente & $87,5(71,9-95,8)$ & $95,8(79,2-100)$ & 0,106 \\
Familiar & $66,7(58,3-79,2)$ & $79,2(58,3-91,7)$ & $\mathbf{0 , 0 1 9}$ \\
Amigos e Apoio Social & $83,3(67,7-91,7)$ & $79,2(66,7-91,7)$ & 0,757 \\
Ambiente Escolar & $66,7(50-83,3)$ & $75(50-100)$ & 0,137 \\
Aspectos Financeiros & $16,7(0-33,3)$ & $8,3(0-25)$ & 0,113 \\
Provocação/Bullying & $62,3(58,9-67,3)$ & $66,2(59,3-71,1)$ & 0,072 \\
Pontuação Global &
\end{tabular}

*Teste de Mann Whitney. Fonte: Autores.

Também foram identificadas diferenças no domínio autopercepção corporal no que se refere ao nível socioeconômico. Verificou-se que adolescentes pertencentes à classe $\mathrm{C} 1$ têm maiores medianas quando comparados com aqueles das demais classes $(\mathrm{p}=0,001)$ (Tabela 2$)$.

Tabela 2 Associação entre QVRS por nível socioeconômico em adolescentes escolares durante o período do isolamento social, 2021.

\begin{tabular}{lllll}
\hline \hline & \multicolumn{4}{l}{ Nível socioeconômico (mediana (Q1 - Q3) } \\
\hline Dimensão & $\mathrm{C} 1(\mathrm{n}=52)$ & $\mathrm{C} 2(\mathrm{n}=26)$ & $\mathrm{D}-\mathrm{E}(\mathrm{n}=9)$ & $\begin{array}{l}\text { valor } \\
\mathrm{p}^{*}\end{array}$ \\
\hline Saúde Atividade Física & $52,5(40-70)$ & $60(35-72,5)$ & $45(35-57,5)$ & 0,491 \\
Autopercepção corporal & $55(40-60) \mathrm{a}$ & $40(38,8-50) \mathrm{b}$ & $40(32,5-50) \mathrm{b}$ & $\mathbf{0 , 0 0 1}$ \\
Autonomia e Tempo Livre & $80(70-95)$ & $80(63,8-86,3)$ & $75(67,5-92,5)$ & 0,77 \\
Sentimentos & $91,7(75-100)$ & $89,6(75-96,9)$ & $75(60,4-91,7)$ & 0,178 \\
Estado Emocional & $27,1(8,3-41,7)$ & $25(12,5-41,7)$ & $41,7(33,3-52,1)$ & 0,117 \\
Família e Ambiente Familiar & $91,7(72,9-95,8)$ & $91,7(78,1-100)$ & $95,8(68,8-100)$ & 0,696 \\
Amigos e Apoio Social & $70,8(58,3-82,3)$ & $66,7(50-87,5)$ & $62,5(52,1-79,2)$ & 0,336 \\
Ambiente Escolar & $83,3(70,8-87,5)$ & $79,2(62,5-92,7)$ & $83,3(58,3-93,8)$ & 0,98 \\
Aspectos Financeiros & $66,7(58,3-91,7)$ & $58,3(39,6-77,1)$ & $66,7(45,8-100)$ & 0,553 \\
Provocação/Bullying & $12,5(0-31,3)$ & $16,7(0-35,4)$ & $8,3(0-25)$ & 0,691 \\
Pontuação Global & $63,7(59,5-68,9)$ & $62,3(58,8-68,6)$ & $60,3(55,6-68,4)$ & 0,445 \\
\hline
\end{tabular}

*Teste de Kruskal Wallis. Fonte: Autores. 
Foram observadas diferenças da QVRS quanto ao consumo alimentar. O consumo de alimentos ultraprocessados pelos adolescentes foi correlacionado com maiores medianas do domínio estado emocional $($ rho $=0,220 ; p=0,042)($ Tabela 3$)$.

Tabela 3 - Associação entre o consumo de alimentos ultraprocessados e QVRS em adolescentes escolares durante o período do isolamento social, 2021.

\begin{tabular}{|c|c|c|}
\hline & & $\begin{array}{l}\text { Alimentos } \\
\text { Ultraprocessados }\end{array}$ \\
\hline \multirow[t]{2}{*}{ Saúde Atividade Física } & $\mathrm{r}$ &, 058 \\
\hline & valor $\mathrm{p}$ &, 593 \\
\hline \multirow[t]{2}{*}{ Autopercepção corporal } & $\mathrm{r}$ &,- 053 \\
\hline & valor $\mathrm{p}$ &, 628 \\
\hline \multirow[t]{2}{*}{ Autonomia e Tempo Livre } & $\mathrm{r}$ &, 148 \\
\hline & valor $\mathrm{p}$ &, 175 \\
\hline \multirow[t]{2}{*}{ Sentimentos } & $\mathrm{r}$ & 031 \\
\hline & valor $\mathrm{p}$ & ,777 \\
\hline \multirow[t]{2}{*}{ Estado Emocional } & $\mathrm{r}$ &, $220^{*}$ \\
\hline & valor $\mathrm{p}$ &, 042 \\
\hline \multirow[t]{2}{*}{ Família e Ambiente Familiar } & $\mathrm{r}$ & ,090 \\
\hline & valor $\mathrm{p}$ & ,410 \\
\hline \multirow[t]{2}{*}{ Amigos Apoio Social } & $\mathrm{r}$ &,- 011 \\
\hline & valor $\mathrm{p}$ & ,923 \\
\hline \multirow[t]{2}{*}{ Ambiente Escolar } & $\mathrm{r}$ &, 156 \\
\hline & valor $\mathrm{p}$ &, 152 \\
\hline \multirow[t]{2}{*}{ Aspectos Financeiros } & $\mathrm{r}$ &, 186 \\
\hline & valor $\mathrm{p}$ & ,086 \\
\hline \multirow[t]{2}{*}{ Provocação/Bullying } & $\mathrm{r}$ &, 001 \\
\hline & valor $\mathrm{p}$ & ,993 \\
\hline \multirow[t]{2}{*}{ Pontuação Global } & $\mathrm{r}$ & ,191 \\
\hline & valor $\mathrm{p}$ &, 078 \\
\hline
\end{tabular}

*Correlação significativa ao nível de 5\%. Fonte: Autores.

A análise multivariada dos fatores associados à QVRS mostrou que o aumento da idade e do IMC influencia na diminuição da QVRS global (Tabela 4).

Tabela 4 - Regressão linear multivariada com a variável estado nutricional e qualidade de vida global em adolescentes escolares durante o período do isolamento social, 2021.

\begin{tabular}{llll} 
Variáveis & Beta & IC 95\% Beta & Valor -p \\
\hline Constante & 98,565 & 73,$145 ; 123,985$ & $<0,001$ \\
Idade & $-1,917$ & $-3,785 ;-0,050$ & 0,044 \\
IMC & $-0,417$ & $-0,807 ;-0,027$ & 0,036 \\
\hline
\end{tabular}




\section{Discussão}

O presente estudo investigou os fatores associados à QVRS em adolescentes no contexto da pandemia de COVID-19 e evidenciou, na análise multivariada, que o aumento do IMC e da idade se associam à diminuição dos escores globais de QVRS, corroborando outros estudos nacionais e internacionais (Muros et al., 2017; Baile et al., 2020; Yi et al., 2019; PeresSousa et al., 2018; D'avila et al., 2019; Liu et al., 2019; Maria, et al., 2017). Uma possível explicação para este resultado consiste no fato de que o excesso de peso na adolescência pode provocar insatisfação com a autoimagem corporal, limitações para a prática de exercícios físicos, além de impactar a saúde mental (Santos et al., 2019; Lima et al., 2018). Muitos adolescentes com excesso de peso podem sofrer estigma relacionado ao peso, discriminação social. Adicionalmente, o aumento do IMC é preditivo da diminuição na QVRS, associando-se ao aumento dos sintomas de depressão (Whitaker et al., 2018). Estas diferenças na QVRS com a idade têm sido justificadas devido às intensas modificações biológicas, sociais e emocionais vivenciadas ao longo da adolescência (Maria, et al., 2017).

Com relação à QVRS e sexo pode-se observar que os meninos apresentaram maiores medianas em comparação às meninas no domínio saúde e atividade física. Este resultado é condizente com o do estudo de Bica e colaboradores (2020), onde meninos apresentaram maiores pontuações nesse domínio. É consenso na literatura que os adolescentes do sexo masculino são mais ativos que as meninas (Santos et al., 2019), provavelmente o que poderia contribuir para maiores escores nesse domínio.

Outro resultado desta pesquisa foi a maior mediana no domínio sentimentos apresentado pelos participantes do sexo masculino, corroborando a publicação de Dantas e Colaboradores (2020). Considerando que o domínio saúde e atividade física também apresentaram medianas maiores em meninos, acredita-se que estes beneficiam-se mais que as meninas das melhorias físicas e mentais proporcionadas pelo exercício físico. É importante destacar que a atividade física se torna uma ferramenta indispensável para o desenvolvimento humano, uma vez que auxilia no bem-estar, contribuindo para a saúde mental e para a proteção cardiovascular (Arruda et al., 2020; Joy, 2020; Lima Junior, 2020).

No que se reportam ao domínio amigos e apoio social, os meninos também apresentaram maiores medianas, igualmente ao estudo de D'avila e colaboradores (2019). Um estudo atual sugere que os adolescentes que possuem uma rede de apoio social têm uma contribuição importante para sua QVRS. No entanto, vários aspectos do suporte social parecem ter diferentes benefícios para meninas e meninos, uma vez que cada um apresenta uma percepção diferente sobre o apoio social. Enquanto para meninos, muitas vezes, a interação com amigos é fundamental, para as meninas, eventualmente e considerando o contexto, o apoio de adultos é mais relevante que de seus pares, impactando, portanto, de forma diferente nos escores de QVRS (Singstad et al., 2021).

As meninas, nessa pesquisa, apresentaram maiores medianas no domínio estado emocional. Jalali-Farahani et al. (2019) encontraram resultado semelhante para a sub escala de funcionamento emocional. Nos estudos de Dantas e colaboradores (2020), D’avila e colaboradores (2019) e Menezes e Colaboradores (2019), este resultado está em desacordo com a presente investigação, pois os meninos apresentaram maior pontuação com relação a este domínio. É importante salientar, no entanto, que as medianas do estado emocional para ambos os sexos foram consideradas inadequadas, baixas. Reforça-se aqui o fato de a coleta de dados ter se dado no contexto da pandemia de COVID-19 que pode ter contribuído para problemas de saúde mental em adolescentes, como depressão, ansiedade e até mesmo luto, em função de perdas de amigos e familiares, o que poderia justificar este achado (Oliveira et al. 2020). Não se pode deixar de reconhecer que a gravidade da doença contribui sobremaneira para impactar a saúde dos participantes, especialmente no que tange aos aspectos relacionados ao isolamento social (Sousa et al,. 2020; Deus \& Coutinho, 2020).

Foi verificado nesse estudo que adolescentes da classe social C1, maior nível socioeconômico identificado entre os participantes, tiveram maiores medianas de autopercepção corporal. Menezes e colaboradores (2019) em sua pesquisa, ao 
avaliarem a QVRS com o nível socioeconômico também encontraram maiores medianas no domínio autopercepção em adolescentes com níveis socioeconômicos mais elevados, o que corrobora os achados da presente investigação. Ossa Gonzáles e colaboradores (2014) avaliaram QVRS, aspectos sociodemográficos, excesso de peso, obesidade e atividade física de adolescentes colombianos e identificaram maiores pontuações em quase todas as dimensões de QVRS nos participantes provenientes de estratos socioeconômicos mais elevados. Os autores afirmam que o maior acesso destas classes às melhores oportunidades para ocupar o tempo livre, realizar atividades físicas e atividades de autocuidado pode refletir em uma percepção mais positiva da QVRS, em especial no domínio autopercepção corporal.

No que se refere ao consumo de alimentos e QVRS, evidenciou-se uma correlação entre maior consumo de alimentos ultraprocessados pelos adolescentes e maiores medianas na dimensão estado emocional. Esse resultado é preocupante, na medida em que um recente estudo de revisão mostra que o consumo alimentar motivado pelo estado emocional e vice-versa está associado ao desenvolvimento e à manutenção do excesso de peso e obesidade (Aparicio et al., 2016).

O padrão de se alimentar como forma de consolo, causando emoções positivas, nostalgia e sentimento de conforto é cada vez mais discutido na literatura e tem sido chamado de comfort food. Esse hábito é frequente em situações de alto estresse emocional, sendo algo negativo para a saúde, uma vez que geralmente os alimentos consumidos são ricos em carboidrato e açúcares. Acredita-se que o alto consumo de comfort food durante o isolamento social, na pandemia de COVID-19, pode estar relacionado com o elevado nível de estresse vivido nesse período (Renzo et al., 2020; Kalarchian et al., 2017). De fato, na quarentena houve um aumento de casos de ansiedade e depressão em adolescentes, os quais sofreram com sentimentos como solidão, incerteza, estresse, medo, frustração e irritabilidade. Além disso, os adolescentes ficaram mais propensos a um estilo de vida sedentário e a uma alimentação pouco nutritiva (Almeida \& da Silva Júnior, 2021).

Com o isolamento social, houve maior permanência dos indivíduos no domicílio, permanecendo expostos a conteúdos de publicidade de alimentos na televisão e na internet, especialmente de alimentos ultraprocessados, os quais corresponderam a 60,7\% dos anúncios (Maia et al., 2017). As propagandas de produtos ultraprocessados apresentam mensagens capazes de induzir um maior consumo desses alimentos por crianças e adolescentes (David et al., 2016). Tais mensagens apresentam estratégias que induzem uma associação do consumo de produtos principalmente ricos em gorduras e açúcar a emoções e aspectos positivos e sedutores, como liberdade, status social, felicidade e até mesmo saúde. Para tal, são apresentadas publicidades com elaboradas técnicas de neuromarketing, fundamentadas na psicologia e neurobiologia a fim de influenciar, de forma sutil, as preferências de alimentação e se consumo (Campagnoli et al., 2015).

Neste estudo, ao avaliar o consumo alimentar pode-se observar que 56,9\% dos adolescentes consumiam de três a cinco alimentos ultraprocessados ao longo do dia. Novamente, considerando o contexto da pandemia de COVID-19, um estudo realizado com adolescentes de cinco países, incluindo o Brasil, observou um aumento no consumo de alimentos fritos e doces, passando de 14\% para 20,7\% durante o confinamento (Ruiz-Roso et al., 2020). A pandemia de COVID-19 no Brasil favoreceu o consumo de alimentos ultraprocessados, pois Raphaelli e colaboradores (2021), reforçando as evidências de mudanças no hábito alimentar da população brasileira durante o período do isolamento. Houve um aumento no consumo de alimentos ultraprocessados, especialmente guloseimas.

A prevalência de excesso de peso está em elevação em todo o mundo e tem aumentando ainda mais devido ao isolamento social. Dados do Sistema de Vigilância Alimentar e Nutricional (SISVAN) referentes ao ano de 2019 apontam que $28,41 \%$ dos adolescentes apresentavam excesso de peso. Em 2020, estes percentuais aumentaram para 30,36\%, refletindo que o excesso de peso está em elevação nesse grupo etário (Ministério da Saúde, 2021). Dados similares referentes ao excesso de peso foram encontrados na presente investigação, onde $32,2 \%$ dos adolescentes avaliados foram classificados com excesso de peso. 
São consolidadas na literatura as implicações do excesso de peso na saúde de adolescentes, tanto do ponto de vista físico quanto emocional (Justino; Ennes; Nucci, 2020). Com o advento da pandemia de Covid-19, estão sendo investigadas as comorbidades e condições clínicas para o agravamento da doença em adolescentes, incluindo o excesso de peso (Frühbeck et al., 2020; Shekerdemian et al., 2020; Zachariah et al., 2020), o que destaca a importância da prevenção e atenção à essa condição.

A presente investigação aponta para a relevância de investimentos em políticas públicas de saúde do adolescente para o combate do excesso de peso, a promoção da alimentação adequada e saudável e a prática de atividade física, uma vez que pode contribuir para a melhoria da QVRS nessa população. São indispensáveis ações junto às famílias e escolas, cenários fundamentais para o fomento da educação em saúde (Nogueira et al., 2020; Testa et al., 2017).

Este estudo possui limitações, por se tratar de um momento pandêmico, não foi possível realizar a aferição dos dados antropométricos, o que levou aos pesquisadores a utilização do peso e estatura autorreferidos. No entanto, essa forma de coleta de dados tem sido utilizada de forma recorrente pelo VIGITEL (Ministério da Saúde, 2020). Além disso, trata-se de um estudo transversal, com limitações relacionadas à temporalidade, além disso, não permitindo inferência de causalidade.

Apesar das limitações da presente pesquisa, destaca-se a importância dessa investigação em plena pandemia de Covid-19, trazendo contribuições para a literatura nesse momento importante em que situa-se o mundo.

\section{Conclusão}

A presente investigação mostrou que o aumento do IMC e da idade contribuem para a diminuição da QVRS global. Sinalizou também diferenças no que se refere ao sexo, nível socioeconômico e consumo de alimentos ultraprocessados. Além disso, é relevante a prevalência de excesso de peso e consumo de alimentos ultraprocessados pelos participantes do estudo.

Esses achados indicam a importância de priorizar investimentos para a promoção da saúde do adolescente, com foco na família e na escola. Investigações longitudinais, de acompanhamento, são indicadas para possibilitar maior clareza na observação do excesso de peso e QVRS em adolescentes.

O estudo possibilitará novas investigações acerca da QVRS no momento pandêmico, favorecendo o reconhecimento dos fatores associados ao declínio da QVRS. Ademais, recomenda-se a utilização de instrumentos que avaliem a frequência alimentar, para um melhor delineamento do consumo alimentar.

\section{Referências}

Almeida, I. M. G., \& da Silva Júnior, A. A. (2021). Os impactos biopsicossociais sofridos pela população infantil durante a pandemia do COVID19. Research, Society and Development, 10(2), e54210212286-e54210212286.

Aparicio, E., Canals, J., Arija, V., Henauw, S. de, \& Michels, N. (2016). The role of emotion regulation in childhood obesity: Implications for prevention and treatment. Nutrition Research Reviews, 29(1), 17-29.

Arruda, E. A., Fonseca, E. da C., Junior, G. R. N., \& Normando, V. M. F. (2020). Impacto da obesidade precoce na qualidade de vida e índices espirométricos de crianças e adolescentes. Revista Eletrônica Acervo Saúde, 12(11), e4836.

Associação Brasileira de Empresas de Pesquisa. (2015). Critério de Classificação Econômica Brasil. São Paulo.

Azambuja Pussieldi, G., de Jesus Silva, F., \& de Almeida Pereira, D. A. (2018). O impacto das aulas de educação física no nível de atividade física, estado de humor e qualidade de vida de adolescentes escolares. Caderno de Educação Física e Esporte, 16(2), 73-83.

Bacchini, D., Licenziati, M. R., Affuso, G., Garrasi, A., Corciulo, N., Driul, D., Tanas, R., Fiumani, P. M., Di Pietro, E., Pesce, S., Crinò, A., Maltoni, G., Iughetti, L., Sartorio, A., Deiana, M., Lombardi, F., \& Valerio, G. (2017). The Interplay among BMI z-Score, Peer Victmization, and Self-Concept in Outpatient Children and Adolescents with Overweight or Obesity. Childhood Obesity, 13(3), 242-249.

Baile, J. I., Guevara, R. M., González-Calderón, M. J., \& Urchaga, J. D. (2020). The relationship between weight status, health-related quality of life, and life satisfaction in a sample of Spanish adolescents. International Journal of Environmental Research and Public Health, $17(9), 3106$.

Bica, I., Pinho, L. M. D., Silva, E. M. B., Aparício, G., Duarte, J., Costa, J., \& Albuquerque, C. (2020). Sociodemographic influence in health-related quality of life in adolescents. ACTA Paulista de Enfermagem, 33, 1-7. 
Campagnoli, R. R., Krutman, L., Vargas, C. D., Lobo, I., Oliveira, J. M., Oliveira, L., Pereira, M. G., David, I. A., \& Volchan, E. (2015). Preparing to caress: A neural signature of social bonding. Frontiers in Psychology, 6(1), 16.

Centro Coordenador do IPAQ no Brasil. (2007). Classificação de atividade física IPAQ. São Paulo.

Collaboration, N. R. F., \& NCD Risk Factor Collaboration. (2017). Worldwide trends in body-mass index, underweight, overweight, and obesity from 1975 to 2016: a pooled analysis of 2416 population-based measurement studies in 128.9 million children, adolescents, and adults. Lancet, $390(10113$ ), $2627-2642$.

Conde, W. L., Mazzeti, C. M. da S., Silva, J. C., Santos, I. K. S. dos, \& Santos, A. M. dos R. (2018). Nutritional status of Brazilian schoolchildren: National Adolescent School-based Health Survey 2015. Revista Brasileira de Epidemiologia, 21(1).

Cunha, L. M. da, Pantoja, M. de S., Portella, M. B., Furlaneto, I. P., \& Lima, A. V. M. (2018). Impacto negativo da obesidade sobre a qualidade de vida de crianças. Revista Brasileira de Obesidade, Nutrição e Emagrecimento, 12(70), 231-238.

Cuschieri, S. (2019). As diretrizes do STROBE. Jornal saudita de anestesia, 13 (Suplemento 1), S31.

D'avila, H. F., Poll, F. A., Reuter, C. P., Burgos, M. S., \& Mello, E. D. (2019). Health-related quality of life in adolescents with excess weight. Jornal de Pediatria, 95(4), 495-501.

Dantas, J. C., Carvalho, D. F. de, Pedraza, D. F., \& Medeiros, C. C. M. (2020). Qualidade de vida relacionada à saúde de adolescentes com excesso de peso. Revista Brasileira de Obesidade, Nutrição e Emagrecimento, 14(85), 241-250.

David, I. A., Krutman, L., Andrade, J. R. de, Araújo, R. L., Braga, F., Gomes, F. da S., Pereira, M. G., Oliveira, L. de, Gleiser, S., Fernández-Santaella, M. C., Delgado, R., \& Volchan, E. (2016). Pistas implícitas e obesidade: estratégias de proteção contra o marketing de alimentos. DEMETRA: Alimentação, Nutrição \& Saúde, 11(2), 383-398

Deus, G. B., \& Coutinho, R. X. (2020). Qualidade de vida durante a pandemia da covid-19: um estudo com estudantes do ensino médio integrado. Revista Interdisciplinar de Ensino, Pesquisa e Extensão, 8(1), 363-373.

Dutra, R. da R., \& Coutinho, R. X. (2020). Comportamentos de risco à saúde: uma análise com estudantes da rede pública de ensino. Semina: Ciências Biológicas e Da Saúde, 41(2), 157.

Frühbeck, G., Baker, J. L., Busetto, L., Dicker, D., Goossens, G. H., Halford, J. C. G., Handjieva-Darlenska, T., Hassapidou, M., Holm, J. C., Lehtinen-Jacks, S., Mullerova, D., O’Malley, G., Sagen, J. V., Rutter, H., Salas, X. R., Woodward, E., Yumuk, V., \& Farpour-Lambert, N. J. (2020). European Association for the Study of Obesity Position Statement on the Global COVID-19 Pandemic. Obesity Facts, 13(2), 292-296.

Gaudenzi, P. (2021). Cenários brasileiros da Saúde Mental em tempos de Covid-19: uma reflexão. Interface-Comunicação, Saúde, Educação, 25, e200330.

Guedes, D. P., \& Guedes, J. E. R. P. (2011). Translation, cross-cultural adaptation and psychometric properties of the KIDSCREEN-52 for the Brazilian population. Revista Paulista de Pediatria, 29(3), 364-371.

Guedes, D. P., \& Mello, E. R. B. (2021). Prevalência de sobrepeso e obesidade em crianças e adolescentes brasileiros: revisão sistemática e metanálise. ABCS Health Sciences, 46, e213.

Gunawardana, S., Gunasinghe, C. B., Harshani, M. S., \& Seneviratne, S. N. (2021). Physical and psychosocial quality of life in children with overweight and obesity from Sri Lanka. BMC Public Health, 21(1), 86.

Instituto Brasileiro de Geografia e Estatística. (2015). Pesquisa Nacional de Saúde do Escolar - 2015. Rio de Janeiro.

Jalali-Farahani, S., Abbasi, B., \& Daniali, M. (2019). Weight associated factors in relation to health-related quality of life (HRQoL) in Iranian adolescents. Health and Quality of Life Outcomes, 17(1), 1-10.

Joy, L. (2020). Staying Active During the Coronavirus Pandemic. Exercise Is Medicine, 1-2.

Justino, M. I., Enes, C. C., \& Nucci, L. B. (2020). Imagem corporal autopercebida e satisfação corporal de adolescentes. Revista Brasileira de Saúde Materno Infantil, 20,715-724.

Kalarchian, M. A., King, W. C., Devlin, M. J., Hinerman, A., Marcus, M. D., Yanovski, S. Z., \& Mitchell, J. E. (2019). Mental disorders and weight change in a prospective study of bariatric surgery patients: 7 years of follow-up. Surgery for Obesity and Related Diseases, 15(5), 739-748.

Lagares, E. B., Resende, K. A., \& Romano, M. C. C. (2019). Obesidade infantil na zona rural: relação com elementos maternos. Rev. enferm. UFPE on line, 796-810.

Lima, LRAD, Teixeira, DM, Martins, PC, Martins, CR, Pelegrini, A., \& Petroski, EL (2018). Imagem corporal e indicadores antropométricos em adolescentes vivendo com HIV. Revista Brasileira de Cineantropometria \& Desempenho Humano, 20, 53-63.

Lima Junior, L. C. (2020). Alimentação saudável e exercícios físicos em meio à pandemia da COVID-19. Boletim de Conjuntura (BOCA), 3(9), 33-41.

Liu, W., Lin, R., Guo, C., Xiong, L., Chen, S., \& Liu, W. (2019). Prevalence of body dissatisfaction and its effects on health-related quality of life among primary school students in Guangzhou, China. BMC Public Health, 19(1), 1-8.

Maia, E. G., Costa, B. V. D. L., Coelho, F. D. S., Guimarães, J. S., Fortaleza, R. G., \& Claro, R. M. (2017). Análise da publicidade televisiva de alimentos no contexto das recomendações do Guia Alimentar para a População Brasileira. Cadernos de Saúde Pública, 33, e00209115.

Maria, A. T., Guimarães, C., Candeias, I., Almeida, S., Figueiredo, C., Pinheiro, A., ... \& Martins, S. (2017). Qualidade de Vida Relacionada com a Saúde em Adolescentes Portugueses: Estudo Numa População Escolar. Acta Pediatr Port, 48(3), 203-11. 
Menezes, M., Lacerda, L. L. V. de, Borella, J., \& Alves, T. P. (2019). Qualidade de Vida e Diabetes Mellitus: Autopercepção de Adolescentes de uma Cidade do Sul do Brasil Quality of Life and Diabetes Mellitus: Perceptions of Teens on a Southern City of Brazil. Psicologia: Teoria e Pesquisa, 35, e35430.

Ministério da Saúde, Secretaria de Atenção à Saúde, \& Departamento de Atenção Básica. (2014). Guia alimentar para a população brasileira. $2^{a}$ ed. Brasília.

Ministério da Saúde. (2021). Secretaria de Atenção Primária à Saúde. Sistema de Vigilância Alimentar e Nutricional. Brasília.

Ministério da Saúde. (2021). Sistema de informação em Saúde para a Atenção Básica. Brasília.

Ministério da Saúde. (2020). Secretaria de Vigilância em Saúde. Departamento de Análise em Saúde e Vigilância de Doenças não Transmissíveis. Brasília.

Muros, J. J., Salvador Pérez, F., Zurita Ortega, F., Gámez Sánchez, V. M., \& Knox, E. (2017). Associação entre comportamentos de estilo de vida saudável e a qualidade de vida relacionada à saúde entre adolescentes ఓ̌r. Jornal de Pediatria, 93, 406-412.

Nascimento, G. B., Schiling, N. D. O., Ubal, S. R., Biaggio, E. P. V., \& Kessler, T. M. (2016). Socio-economic classification and quality of life of family members of children and teenagers with hearing disability. Revista CEFAC, 18, 657-666.

Nogueira, E., Nascimento, F. de A. do, Souza, R. L. de, \& Silva, W. M. da. (2020). A obesidade infantil no Brasil e fatores associados: desafios para os professores de educação física - Dialnet. Revista Internacional de Apoyo a La Inclusión, Logopedia, Sociedad y Multiculturalidad, 6(1), 13-24.

Oliveira, W. A. D., Silva, J. L. D., Andrade, A. L. M., Micheli, D. D., Carlos, D. M., \& Silva, M. A. I. (2020). A saúde do adolescente em tempos da COVID19: scoping review. Cadernos de Saúde Pública, 36.

Ossa González, E. J., Arango Vélez, E. F., Rodríguez, C. V., Contreras, A. M., Estrada Restrepo, A., Bedoya Berrío, G., Ochoa, G. A. (2014). Calidad de vida relacionada con la salud en un grupo de adolescentes de Medellín (Colombia): Asociación con aspectos sociodemográficos, exceso de peso u obesidad y actividad física. Revista Colombiana de Psicología, 23(2), 255-267.

Otto, C., Haller, A. C., Klasen, F., Hölling, H., Bullinger, M., Ravens-Sieberer, U., \& BELLA Study Group. (2017). Risk and protective factors of healthrelated quality of life in children and adolescents: results of the longitudinal BELLA study. PLoS One, 12(12), e0190363.

Perez-Sousa, M. A., Olivares, P. R., Escobar-Alvarez, J. A., Parraça, J. A., \& Gusi, N. (2018). Fitness as mediator between weight status and dimensions of health-related quality of life. Health and Quality of Life Outcomes, 16(1), 155.

Rankin, J., Matthews, L., Cobley, S., Han, A., Sanders, R., Wiltshire, H. D., \& Baker, J. S. (2016). Psychological consequences of childhood obesity: psychiatric comorbidity and prevention. Adolescent health, medicine and therapeutics, 7, 125.

Raphaelli, C. O., Figueiredo, M. F. de, Pereira, E. dos S., \& Granada, G. G. (2021). A pandemia de COVID-19 no Brasil favoreceu o consumo de alimentos ultraprocessados? Brazilian Applied Science Review, 5(3), 1297-1313.

Renzo, L., Gualtieri, P., Cinelli, G., Bigioni, G., Soldati, L., Attinà, A., \& De Lorenzo, A. (2020). Aspectos psicológicos e hábitos alimentares durante o confinamento domiciliar COVID-19: resultados da pesquisa online italiana EHLC-COVID-19. Nutrients, 12 (7), 2152.

Ruiz-Roso, M. B., Padilha, P. de C., Mantilla-Escalante, D. C., Ulloa, N., Brun, P., Acevedo-Correa, D., Peres, W. A. F., Martorell, M., Aires, M. T., Cardoso, L. de O., Carrasco-Marín, F., Paternina-Sierra, K., Rodriguez-Meza, J. E., Montero, P. M., Bernabè, G., Pauletto, A., Taci, X., Visioli, F., \& Dávalos, A (2020). Covid-19 confinement and changes of adolescent's dietary trends in Italy, Spain, Chile, Colombia and Brazil. Nutrients, 12(6), 1-18.

Santos, J. P. D., Mendonça, J. G. R., Barba, C. H. D., Carvalho, J. J. D., Bernaldino, E. D. S., Farias, E. D. S., \& Souza, O. F. D. (2019). Fatores associados a não participação nas aulas de educação física escolar em adolescentes. Journal of Physical Education, 30.

Sattamini, I. F. (2019). Instrumentos de avaliação da qualidade de dietas: desenvolvimento, adaptação e validação no Brasil [Tese]. Universidade de São Paulo. São Paulo.

Shekerdemian, L. S., Mahmood, N. R., Wolfe, K. K., Riggs, B. J., Ross, C. E., McKiernan, C. A., Heidemann, S. M., Kleinman, L. C., Sen, A. I., Hall, M. W., Priestley, M. A., McGuire, J. K., Boukas, K., Sharron, M. P., \& Burns, J. P. (2020). Characteristics and outcomes of children with coronavirus disease 2019 (COVID-19) infection admitted to US and Canadian pediatric intensive care units. JAMA Pediatrics, 174(9), 868-873.

Singstad, M. T., Wallander, J. L., Greger, H. K., Lydersen, S., \& Kayed, N. S. (2021). Perceived social support and quality of life among adolescents in residential youth care: a cross-sectional study. Health and Quality of Life Outcomes, 19(1), 1-12.

Sousa, G. C., Lopes, C. S. D., Miranda, M. C., da Silva, V. A. A., \& Guimarães, P. R. (2020). A Pandemia de COVID-19 e suas repercussões na epidemia da obesidade de crianças e adolescentes. Revista Eletrônica Acervo Saúde, 12(12), e4743-e4743.

Testa, W. L., Poeta, L. S., \& Duarte, M. de F. da S. (2017). Exercício físico com atividades recreativas: uma alternativa para o tratamento da obesidade infantil. Revista Brasileira de Obesidade, Nutrição e Emagrecimento, 11(62), 49-55.

Vallandro, J. P., \& Dalpubel, V. (2015). Imagem corporal e sua relação com o estado nutricional e a qualidade de vida de adolescentes de um município do interior do Rio Grande do Sul. Nutrire, 40(1), 1-9.

Whitaker, B. N., Fisher, P. L., Jambhekar, S., Com, G., Razzaq, S., Thompson, J. E., ... \& Ward, W. L. (2018). Impact of degree of obesity on sleep, quality of life, and depression in youth. Journal of Pediatric Health Care, 32(2), e37-e44.

Yi, X., Fu, Y., Burns, R., \& Ding, M. (2019). Weight status, physical fitness, and health-related quality of life among Chinese adolescents: A cross-sectional study. International Journal of Environmental Research and Public Health, 16(13), 2271.

Zachariah, P., Johnson, C. L., Halabi, K. C., Ahn, D., Sen, A. I., Fischer, A., Banker, S. L., Giordano, M., Manice, C. S., Diamond, R., Sewell, T. B., Schweickert, A. J., Babineau, J. R., Carter, R. C., Fenster, D. B., Orange, J. S., McCann, T. A., Kernie, S. G., \& Saiman, L. (2020). Epidemiology, Clinical Features, and Disease Severity in Patients with Coronavirus Disease 2019 (COVID-19) in a Children's Hospital in New York City, New York. JAMA 
Research, Society and Development, v. 10, n. 13, e181101320974, 2021

(CC BY 4.0) | ISSN 2525-3409 | DOI: http://dx.doi.org/10.33448/rsd-v10i13.20974

Pediatrics, 174(10), e202430.

Zhao, Z., Ma, Y., Han, Y., Liu, Y., Yang, K., Zhen, S., \& Wen, D. (2018). Psychosocial correlates of food addiction and its association with quality of life in a non-clinical adolescent sample. Nutrients, 10(7), 837. Colocar espaço entre uma referência e outra. Lembre-se que usamos a norma APA. (fonte TNR 8 espaço simples -justificado) 